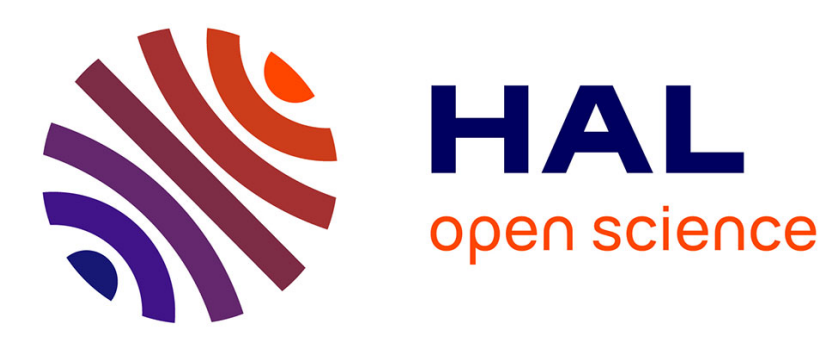

\title{
Experimental characterization of transition region in rotating-disk boundary layer
}

Muhammad Ehtisham Siddiqui, Vasudevan Mukund, Julian Scott, Benoît Pier

\section{To cite this version:}

Muhammad Ehtisham Siddiqui, Vasudevan Mukund, Julian Scott, Benoît Pier. Experimental characterization of transition region in rotating-disk boundary layer. Physics of Fluids, 2013, 25, pp.034102. 10.1063/1.4798435 . hal-00806379

\section{HAL Id: hal-00806379 \\ https://hal.science/hal-00806379}

Submitted on 2 Apr 2013

HAL is a multi-disciplinary open access archive for the deposit and dissemination of scientific research documents, whether they are published or not. The documents may come from teaching and research institutions in France or abroad, or from public or private research centers.
L'archive ouverte pluridisciplinaire HAL, est destinée au dépôt et à la diffusion de documents scientifiques de niveau recherche, publiés ou non, émanant des établissements d'enseignement et de recherche français ou étrangers, des laboratoires publics ou privés. 


\title{
Experimental characterization of transition region in rotating-disk boundary layer
}

\author{
M. E. Siddiqui, ${ }^{1,2}$ V. Mukund, ${ }^{1,3}$ J. Scott, ${ }^{1}$ and B. Pier ${ }^{1, a)}$ \\ ${ }^{1}$ Laboratoire de mécanique des fluides et d'acoustique, École centrale de Lyon, CNRS, \\ Université Claude-Bernard Lyon 1, INSA, 36 avenue Guy-de-Collongue, \\ 69134 Écully cedex, France \\ ${ }^{2}$ Ghulam Ishaq Khan Institute of Engineering Sciences and Technology, Topi, Swabi, Pakistan \\ ${ }^{3}$ Max Planck Institute for Dynamics and Self-Organization, 37077 Göttingen, Germany
}

(Received 12 October 2012; accepted 6 March 2013; published online 29 March 2013)

\begin{abstract}
The three-dimensional boundary layer due to a disk rotating in otherwise still fluid is well known for its sudden transition from a laminar to a turbulent regime, the location of which closely coincides with the onset of local absolute instability. The present experimental investigation focuses on the region around transition and analyses in detail the features that lead from the unperturbed boundary layer to a fully turbulent flow. Mean velocity profiles and high-resolution spectra are obtained by constant-temperature hot-wire anemometry. By carefully analysing these measurements, regions in the flow are identified that correspond to linear, weakly nonlinear, or turbulent dynamics. The frequency that dominates the flow prior to transition is explained in terms of spatial growth rates, derived from the exact linear dispersion relation. In the weakly nonlinear region, up to six clearly identifiable harmonic peaks are found. High-resolution spectra reveal the existence of discrete frequency components that are deemed to correspond to fluctuations stationary with respect to the disk surface. These discrete components are only found in the weakly nonlinear region. By systematically acquiring low- and high-resolution spectra over a range of narrowly spaced radial and axial positions, it is shown that while the transition from laminar to turbulent regimes occurs sharply at some distance from the disk surface, a complex weakly nonlinear region of considerable radial extent continues to prevail close to the disk surface. (ㅇ) 2013 American Institute of Physics. [http://dx.doi.org/10.1063/1.4798435]
\end{abstract}

\section{INTRODUCTION}

Three-dimensional boundary layers are characterized by a rapid variation of all three velocity components in the neighbourhood of a solid surface. This happens whenever a body is rotating with respect to the surrounding fluid or if a flow impacts an obstacle obliquely. Practical configurations include the flow around compressor blades, aircraft wings, ship hulls, or wind turbines. All these configurations are prone to strong instabilities that rapidly lead to turbulence. The objective of the present investigation is to carry out detailed measurements of the dynamics of such a flow in order to shed new light on the mechanisms governing the complex transition scenario that leads from a laminar to a turbulent state.

Within the class of three-dimensional boundary layers, the flow with the simplest geometry is probably that due to a large disk rotating in otherwise still fluid: von Kármán ${ }^{1}$ obtained the basic flow as an exact similarity solution of the Navier-Stokes equations. This basic-flow solution, illustrated

\footnotetext{
a)Electronic mail: benoit.pier@ec-lyon.fr
} 




FIG. 1. Basic flow over rotating disk.

in Figure 1, displays a constant boundary layer thickness proportional to

$$
\delta=\sqrt{\frac{\nu}{\Omega}}
$$

where $v$ is the kinematic viscosity and $\Omega$ the disk rotation rate. Note that the azimuthal velocity drops to about $1 \%$ of the disk velocity at a distance $5 \delta$ from the disk surface. Throughout this study, the axial coordinate $Z$ and radial coordinate $R$ are non-dimensionalized by $\delta$. The radial, azimuthal, and axial basic flow velocity components, non-dimensionalized by $\Omega \delta$, are

$$
U_{r}=R U(Z) \quad \text { and } \quad U_{\theta}=R V(Z) \quad \text { and } \quad U_{z}=W(Z)
$$

where $U(Z), V(Z)$, and $W(Z)$ are the von Kármán similarity profiles.

When unstable, this boundary layer develops cross-flow vortices, as observed experimentally by Gregory et al. ${ }^{2}$ Transition from laminar to turbulent regimes occurs at a non-dimensional radius in the range $500-550 .^{2-7}$ Lingwood $^{8}$ has found that this boundary layer undergoes transition from convective to absolute instability at a critical radius $R^{c a}=507$ that closely corresponds to the position of experimentally observed transition to turbulence. However, it has been shown by Davies and Carpenter ${ }^{9}$ that, within a strictly linear framework, this flow is globally stable despite the presence of an extended region of local absolute instability. Subsequently, a fully nonlinear analysis ${ }^{10}$ led to a consistent theory able to account for the onset of self-sustained finite-amplitude fluctuations beyond $R^{c a}$. While all the previous theoretical analyses assume a rotating disk of infinite extent, Healey ${ }^{11}$ has recently been able to take into account the effect of the outer edge of the disk, study its influence on the global instability, and provide a possible explanation for the scatter in transition locations reported by different experimental studies.

Despite numerous investigations, it appears that the precise mechanism leading from laminar to turbulent states is not yet fully understood. The present experimental investigation has been undertaken to precisely map out the near transition region by determining how mean-flow deviations from the von Kármán solution and the spectral content of the fluctuations vary with radial and axial positions, with special attention to the near-disk region. A similar aim, but with a different approach, has been independently pursued by Imayama $e t$ al. ${ }^{12}$ The present paper is based on Ref. 13 and a preliminary account of our findings was given in Ref. 14.

The structure of the manuscript is the following. The experimental arrangement is described in Sec. II. Then we describe our experimental observations of mean velocity profiles (Sec. III A), low-resolution spectra (Sec. III B 1), and high-resolution spectra (Sec. III B 2). These results are discussed in detail in Sec. IV. 


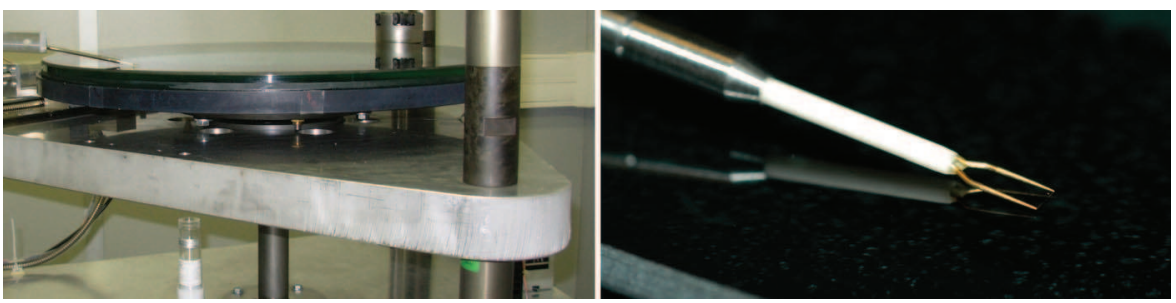

FIG. 2. Experimental setup. (Left) $50 \mathrm{~cm}$ diameter glass disk that may be rotated up to $1500 \mathrm{rpm}$. (Right) Hot-wire probe positioned parallel to the disk surface and aligned in the radial direction, positioned by a high-precision two-axes mechanism.

\section{EXPERIMENTAL ARRANGEMENT}

The rotating-disk facility designed for the present investigation (Figure 2) consists of a glass disk of $500 \mathrm{~mm}$ in diameter that is rotated at constant angular velocity $\Omega$, up to $1500 \mathrm{rpm}$. Details of the experimental arrangement are given in Ref. 13. The disk surface was measured by a linear variable differential transducer (LVDT) and its position adjusted so as to achieve best alignment with a plane normal to the rotation axis. The disk roughness, characterized by the arithmetic average of absolute surface values, was measured as $1.6 \mu \mathrm{m}$. A high-precision two-axes traversing mechanism was used for positioning of a hot-wire probe, with radial and axial precisions of $20 \mu \mathrm{m}$ and $2 \mu \mathrm{m}$, respectively.

For the local velocity measurements, constant-temperature hot-wire anemometry is used, being particularly suitable for the measurement of flows with very fast fluctuations at a fixed point in space. A single Dantec hot wire of type 55P01 is used, consisting of a $5 \mu \mathrm{m}$ diameter and $3 \mathrm{~mm}$ wide platinum-plated tungsten wire with a $1.25 \mathrm{~mm}$ flow sensitive length at its center. The hot wire is positioned parallel to the disk surface and aligned in the radial direction so as to measure the azimuthal flow component. The traversing mechanism and velocity measurements are controlled and recorded by a dedicated computer.

The hot wire must be calibrated using known flow velocities. Rather than using a separate wind tunnel, which would require frequent removal of the probe from its support, we always calibrated the hot-wire probe against the laminar boundary-layer profile, given by the von Kármán solution. Knowing the disk rotation rate, the distance of the hot wire from the disk surface and from the disk axis, the boundary-layer velocity at the hot-wire position can be calculated. The hot wire is then calibrated by measuring the mean output voltage from the anemometer for a range of flow speeds. In this calibration process, the disk rotation rate and position of the probe are chosen to remain well within the laminar region. A fourth-order polynomial was used to fit the velocity-voltage data pairs, and this polynomial subsequently used to convert measured instantaneous voltages to flow velocities. Keeping in mind that the hot-wire anemometry has a sensitive temperature dependence, this calibration was repeated before each measurement. Data acquisition is performed by moving the hot wire in the radial and axial directions for a constant disk speed. This process is then repeated for different values of the disk speed, and, after non-dimensionalization, the data acquired at different rotation rates collapse to the same values. See Ref. 13 for details of the experimental procedure.

\section{RESULTS}

\section{A. Mean velocity profiles}

The main focus of this paper is the spectral analysis of velocity time-series. However, the measured mean velocity profiles are briefly presented first for completeness and validation of the setup.

Mean azimuthal velocity components are shown in Figure 3 over the range $350 \leq R \leq 600$. In these plots, velocities are normalized by the velocity of the disk surface at the corresponding radial position. 


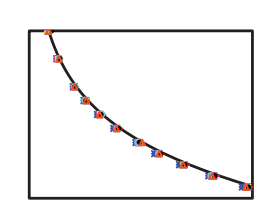

(a)
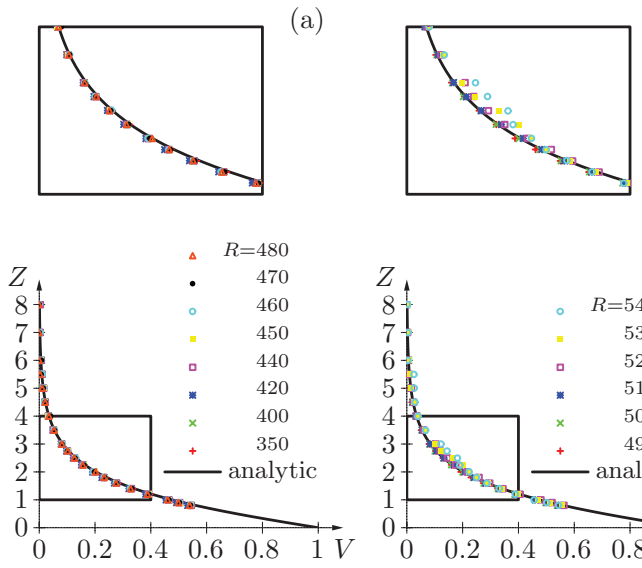

(b)

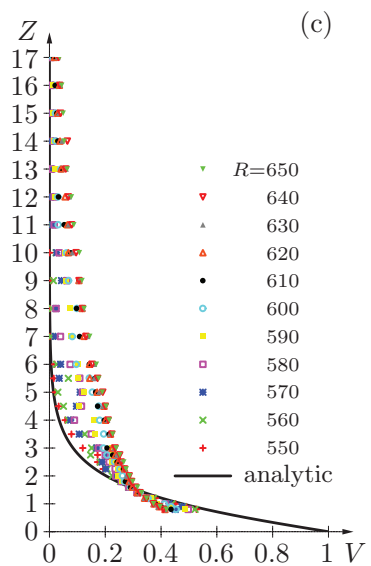

FIG. 3. Azimuthal mean-flow velocity profiles for non-dimensional radii in the range $350 \leq R \leq 650$, along with the analytical profile (solid line); insets show magnified view of region from $Z=1$ to $Z=4$. (a) Measured profiles closely follow the analytical curve for $R \leq 480$. (b) Small deviations of the measured profile from the analytical curve are observed for $2 \leq Z \leq 3$ and $490 \leq R \leq 540$. (c) For $R \geq 550$, strong mean-flow corrections, extending beyond $Z=15$ by $R=650$, characterize a transitional and fully turbulent boundary layer.

At low values of $R$, the measured profiles very closely follow the von Kármán similarity profile, see Figure 3(a).

For $490 \leq R \leq 540$, small but significant differences between the measured profiles and the analytical profile are observed. Figure 3(b) shows that these mean flow corrections are confined to a narrow region of the boundary layer $(2 \leq Z \leq 3)$ and their amplitude is less than $5 \%$ of the maximum velocity in this range. In the convectively unstable range, $284<R<507$, they can be interpreted as reflecting the radial growth of instabilities (cross-flow vortex modes) in the boundary layer. The confinement in $Z$ is consistent with the structure of the unstable-mode eigenfunctions, as computed, e.g., in Ref. 10 .

For $R \geq 550$, stronger mean-flow distortions are observed, which progressively extend beyond $Z=15$, see Figure 3(c). Such boundary-layer thickening is the characteristic of the development of turbulent boundary layers. This behaviour of the mean-flow profiles corresponds exactly to what has been obtained in previous studies ${ }^{6,7}$ and is thus deemed a sufficient validation of the present experimental setup.

\section{B. Spectral analysis}

Frequency contents of disturbances in the boundary-layer are investigated by calculating Fourier power spectra from azimuthal-velocity time series at different non-dimensional radii $R$ and disk normal positions $Z$.

For most of the previously published experimental results, power spectra have a low frequency resolution, of the order of $\Delta \omega=1$, non-dimensionalized by the disk rotation rate. In order to obtain high-resolution spectra, the velocity signals were recorded over long time intervals, typically a few thousand revolutions of the disk.

In post-processing, the power spectra are obtained by Fourier analyses of these signals. The procedure is the following. The signal is split into $n$ series covering $m$ disk revolutions each. Each of the $n$ series is Fourier analysed and the squared moduli of the complex Fourier amplitudes are then averaged over the $n$ spectra. By varying $m$, spectra of different frequency resolutions are obtained. Typical "low-resolution" spectra are obtained with $m=1$, while spectra obtained with $m=100$ or $m$ $=1000$ are referred to as high-resolution spectra, with resolution $\Delta \omega=0.01$ or 0.001 , respectively. Thus, spectra with different resolutions can be obtained from the same data, shedding new light on the frequency content of the fluctuations developing in the rotating-disk boundary layer. 


\section{Low-resolution spectra}

Low-resolution power spectra are shown in Figure 4. At low values of $R$, these mainly consist of uniform background noise. Starting at $R=450$, a peak with a maximum near $\omega=30$ emerges. This is an indication of the development of growing cross-flow modes with a characteristic frequency around 30, but these modes as yet cause no significant distortion of the mean flow: as shown in Sec. III A, for $R \leq 480$, the measured mean-flow profiles closely follow the von Kármán solution.

At higher $R$, power spectra show the progressive growth and development of the disturbance. A second-harmonic peak appears for $R=490$ (Figure 4(b)), indicating effects of nonlinear interactions. At even larger values of $R$, higher harmonics of the fundamental peak arise near $\omega=60,90,120$, 150 , and 180 . Note that the harmonics first appear at different values of $R$ depending on the distance $Z$ from the disk surface. This will be further discussed in Sec. IV.

At and above $R=520$, Figures 4(e)-4(h) show the progressive increase of a broadband spectral component, followed by the disappearance of the modal peaks. This corresponds to transition to turbulence. By $R=600$ (Figure 4(h)), there are no longer any clear peaks associated with the modes and the flow is fully turbulent throughout the boundary layer.
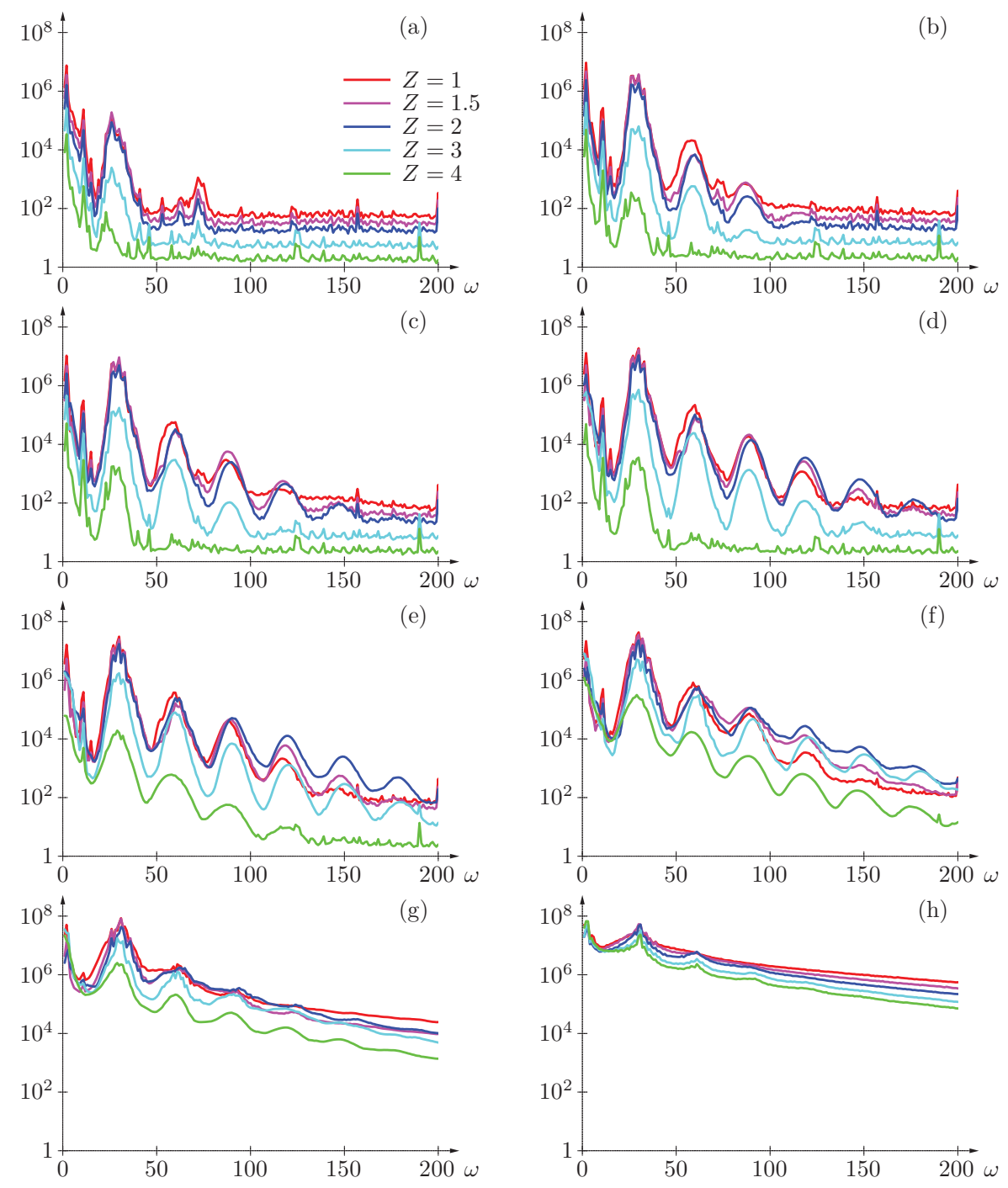

FIG. 4. Low-resolution spectra for (a) $R=450$, (b) $R=490$, (c) $R=500$, (d) $R=510$, (e) $R=520$, (f) $R=530$, (g) $R=550$, and (h) $R=600$. Spectral amplitudes are plotted on a logarithmic scale for disk normal positions $Z=1,1.5,2,3$, and 4 . 

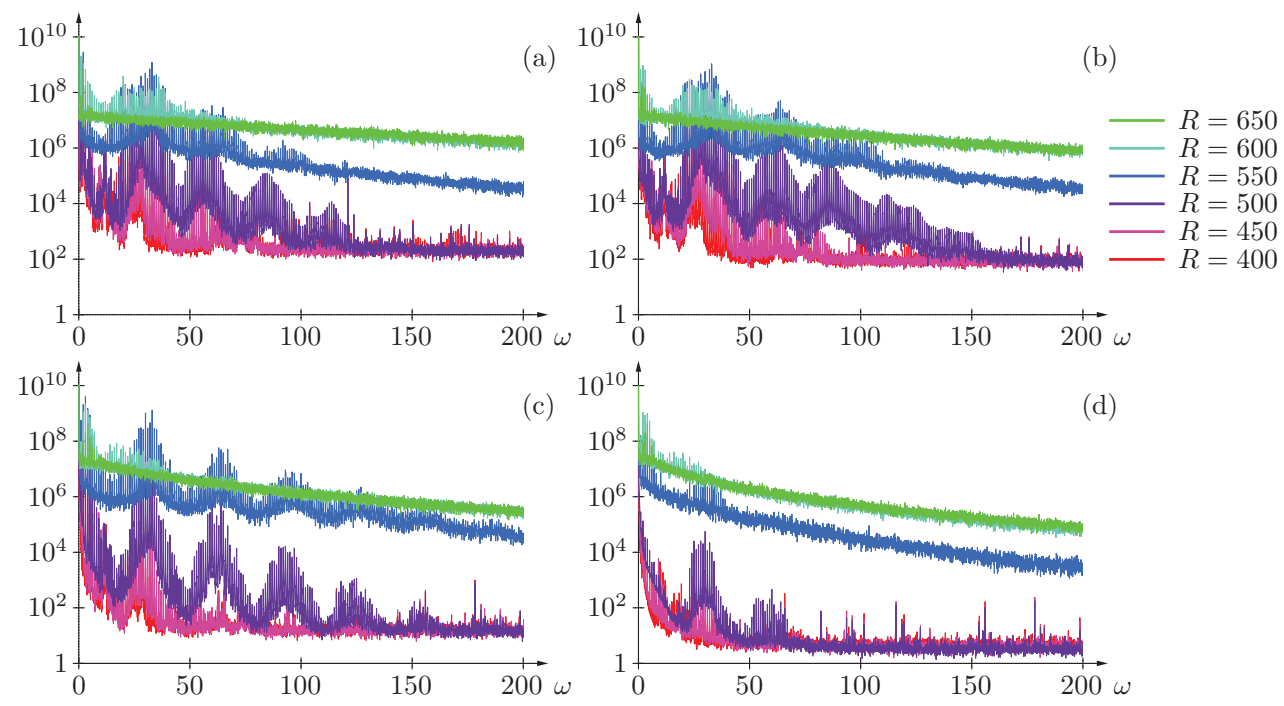

FIG. 5. High-resolution spectra corresponding to $R=400,450, \ldots, 650$ and (a) $Z=0.8$, (b) $Z=1.5$, (c) $Z=3.0$, and (d) $Z=6.0$

From these low-resolution spectra, three distinct flow regimes can be identified: a linear regime where the boundary layer only displays small-amplitude perturbations associated with at most a single peak in the spectrum; a weakly nonlinear regime characterized by a dominant frequency and its harmonics due to nonlinear interactions; and a fully turbulent regime where the harmonic peaks have been superseded by a continuous spectrum.

In terms of mean velocities, these three regimes broadly correspond to no, weak, and strong mean-flow deviations from the similarity profile, as shown in Sec. III A. A complete characterization of the different regions of the rotating-disk boundary layer will be discussed in Sec. IV.

\section{High-resolution spectra}

As described earlier, high-resolution spectra are derived by Fourier analyzing the velocity timeseries recorded over long temporal intervals. Plots shown in Figure 5 have a frequency resolution of $\Delta \omega=0.01$ and are obtained by averaging 50 spectra computed over 100 disk revolutions. Spectra obtained with a frequency resolution of $\Delta \omega=0.001$ look very similar and are not shown here. Overall, the high-resolution spectra display similar characteristics to their low-resolution counterparts, but a distinctive feature is the existence of discrete peaks at integer multiples of the disk rotation rate. These peaks are smoothed out in the low-resolution spectra. The discrete peaks are more clearly visible in the close-up views shown in Figure 6. The radial evolution of high-resolution spectra in the range $450 \leq R \leq 650$ for $Z=1$ and $Z=6$ is illustrated in Figure 7 for $20<\omega<40$.

These measurements show that the velocity spectra are made up of both a continuous and a discrete part. The discrete part corresponds to flow components that have exactly the same periodicity as the disk.

It is observed that both the discrete and continuous parts of the spectrum grow with $R$. However, the discrete component is the dominant feature only in the "weakly nonlinear" regime, while it is hardly developed in both the laminar and in the turbulent regions. Also, it is more prominent for the most amplified frequency, around $\omega \simeq 30$, and its harmonics.

At $R=350$, the spectrum is essentially background noise and the discrete component appears to be irrelevant. For, say, $450<R<510$, the discrete part grows to form a peak around $\omega \simeq 30$. This growth continues up to $R=530$, but involves a wider band of frequencies and then saturates. From $R=530$ on, the continuous part grows as the boundary layer approaches the turbulent regime. By $R=650$, the spectrum corresponds to a fully turbulent flow without any visible discrete component. 

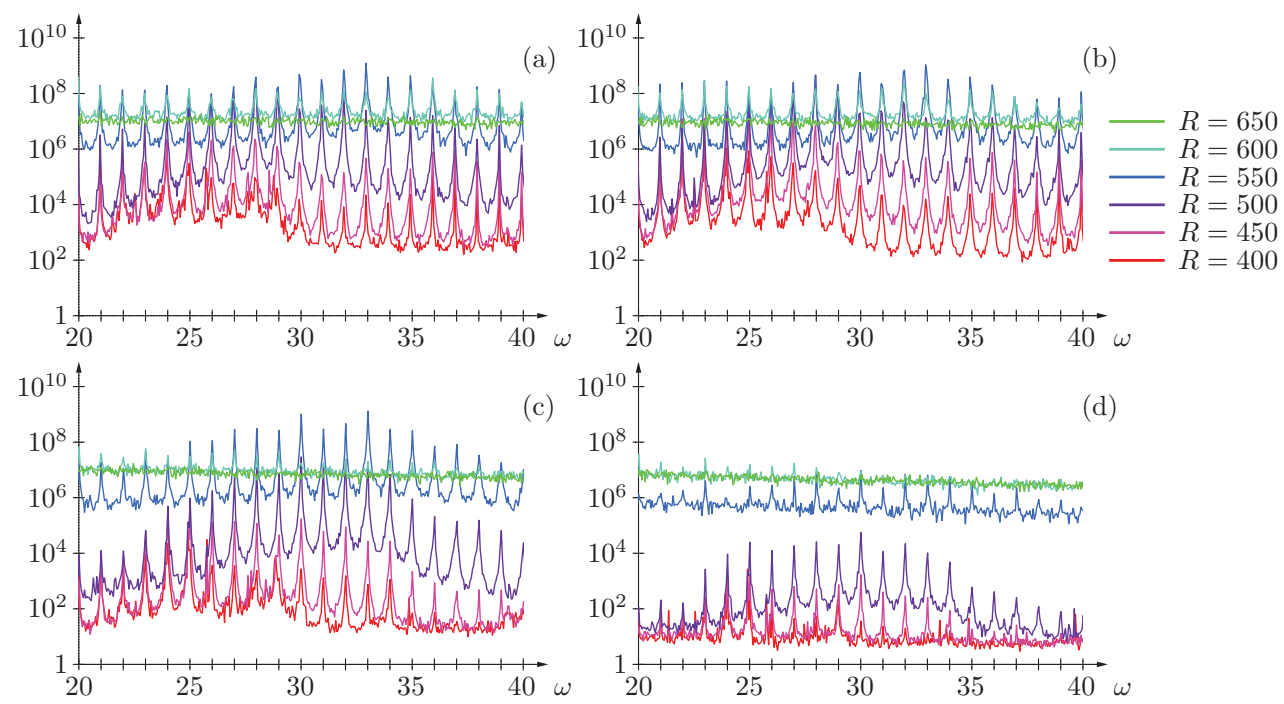

FIG. 6. Closeup view for $20<\omega<40$ of high-resolution spectra corresponding to $R=400,450, \ldots, 650$ and (a) $Z=0.8$, (b) $Z=1.5$, (c) $Z=3.0$, and (d) $Z=6.0$.

\section{DISCUSSION}

In the present experimental investigation of a rotating-disk flow, hot-wire velocity measurements have been carried out to characterize the dynamics of this boundary layer with special emphasis on the near transition region.

As expected, mean velocity profiles follow the self-similar von Kármán solution at low radii, small but significant mean-flow deviations from the similarity profile are obtained for $490 \leq R \leq 540$ and $2 \leq Z \leq 3$, and fully turbulent profiles prevail for $R \geq 550$, characterized by strong mean-flow corrections extending beyond $Z=15$.

Spectral analyses of velocity-series yield the frequency content of the perturbations that develop in the different regions of the boundary layer. Low-resolution spectra show that the first perturbations to develop with increasing $R$ exhibit characteristic frequencies near $\omega=30$. This corresponds to the largest spatial growth rate for perturbations that are stationary with respect to the disk. Figure 8 shows the spatial growth rate $-\alpha_{i}$ as function of frequency $\omega$ and azimuthal mode number $\beta$, derived from the local linear dispersion relation $\omega=\Omega(\alpha, \beta ; R)$. This dispersion relation is obtained by considering perturbations of the form $\operatorname{expi}(\alpha r+\beta \theta-\omega t)$ and numerically implementing a complete stability analysis based on the linearized Navier-Stokes equations, as in Ref. 10. The plot in Figure 8, shows that at $R=500$ the largest spatial growth rate $-\alpha_{i} \simeq 0.1$ is obtained for $(\omega, \beta) \simeq(47,62)$. However, perturbations with $\omega \neq \beta$ are traveling with respect to the disk surface. Considering that fluctations measured in the convectively unstable range $280<R<510$ are mainly

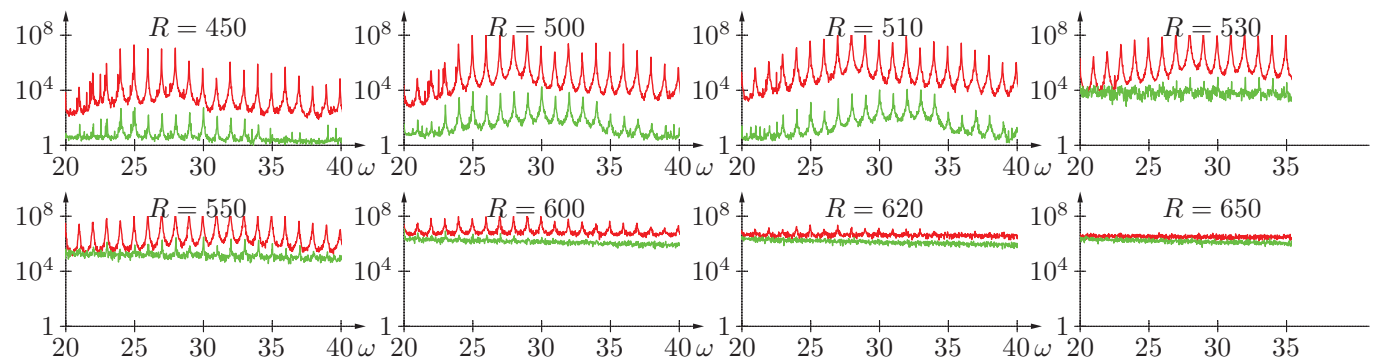

FIG. 7. Radial evolution of high-resolution power spectra in the range $20 \leq \omega \leq 40$, for $450 \leq R \leq 650$ and $Z=1$ (upper curves) and $Z=6$ (lower curves). 


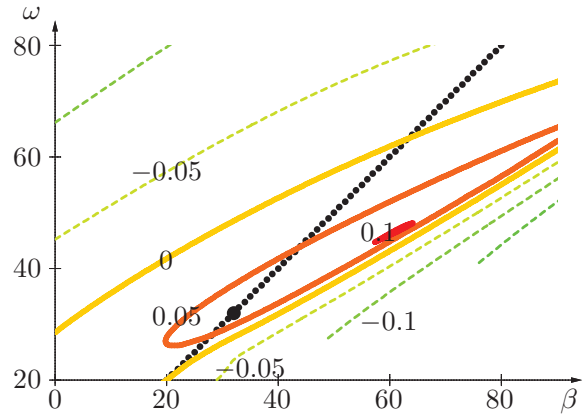

FIG. 8. Isolines of spatial growth rates $-\alpha_{i}$ at $R=500$, derived from the exact local linear dispersion relation $\omega=\Omega(\alpha, \beta$; $R$ ), in the $\omega-\beta$ (frequency-azimuthal mode number) plane.

due to imperfections and roughness of the disk surface and are therefore stationary with respect to the disk, they correspond to the line $\omega=\beta$ in Figure 8. For these waves rotating at the same rate as the disk, maximum spatial growth is attained with $\omega=\beta=32$ (at $R=500$ ), indicated by a big dot in Figure 8. It is also found that this maximally amplified frequency hardly depends on $R$ in the convectively unstable region. Thus, by carrying out a systematic computation of the spatio-temporal dispersion relation based on the linearized Navier-Stokes equations, we confirm the origin of the fundamental harmonic peak observed in the spectra from onset of linear instability to transition to turbulence.

With increasing radial distance, the flow enters a weakly nonlinear regime, characterized by a harmonic spectrum made up of the dominant fundamental frequency and its harmonics. Eventually, the boundary layer enters a fully turbulent state associated with the disappearance of the modal peaks, replaced by a broad continuous spectrum. The detailed characteristics of these spectra, such as the number of harmonic peaks that may be identified or the occurrence of a broad spectrum, depend both on the radial and the axial locations of the measurement. Counting of the harmonic peaks can be performed automatically by a post-processing script: for each of the low-resolution spectra (cf. Figure 4), the script first extracts the maxima located approximately at multiples of the fundamental frequency and then retains those that are separated by a sufficiently deep minimum. A detailed map of the flow structures is given in Figure 9(a). Measurements have been systematically carried out for $R$ $=350,360, \ldots, 650$ and $Z=0.8,1.0,1.5,2.0,3.0,4.0$, and 6.0. For each of these locations, the symbol in Figure 9(a) depicts the type of spectrum that is obtained: the number of circles corresponds to the number of harmonic peaks that could be identified and a grey background indicates the presence of a broadband component. A single circle on white background thus corresponds to a laminar boundary layer that is at most linearly perturbed. The weakly nonlinear region corresponds to at least two identifiable broad harmonic peaks and thus starts with the double-circle symbols. With increasing radial distance, higher-order harmonics develop; up to six clearly identifiable harmonic peaks have been found at $Z=3$ and $510 \leq R \leq 520$. Then, a broadband component appears near $R=530$ (symbols with grey background) that progressively replaces the harmonic spectrum. Eventually a fully turbulent regime is reached with no identifiable harmonic peaks in the spectrum. From this map one sees that the transition from laminar to turbulent flow is relatively sharp far from the disk surface (say $Z>5$ ), while a nonlinear region of considerable extent is observed in the near-disk region. This nonlinear region can be considered to start as early as $R \simeq 450$ and to survive up to $R \simeq 570$, well beyond a single transition location near $R \simeq 530$.

Velocity spectra with high frequency resolution have been obtained from velocity signals recorded over long time-periods. These high-resolution spectra reveal the existence of narrow peaks located at integer values of the frequency. Since these discrete peaks appear every integer multiple of disk rotation rate, they are associated with the flow components that have the same periodicity as the disk and that are probably stationary with respect to the disk surface. It should be noted that these discrete peaks are not an experimental artifact, for otherwise they would also be present at low and high values of $R$. In order to map out the regions where this discrete part of the spectrum is 


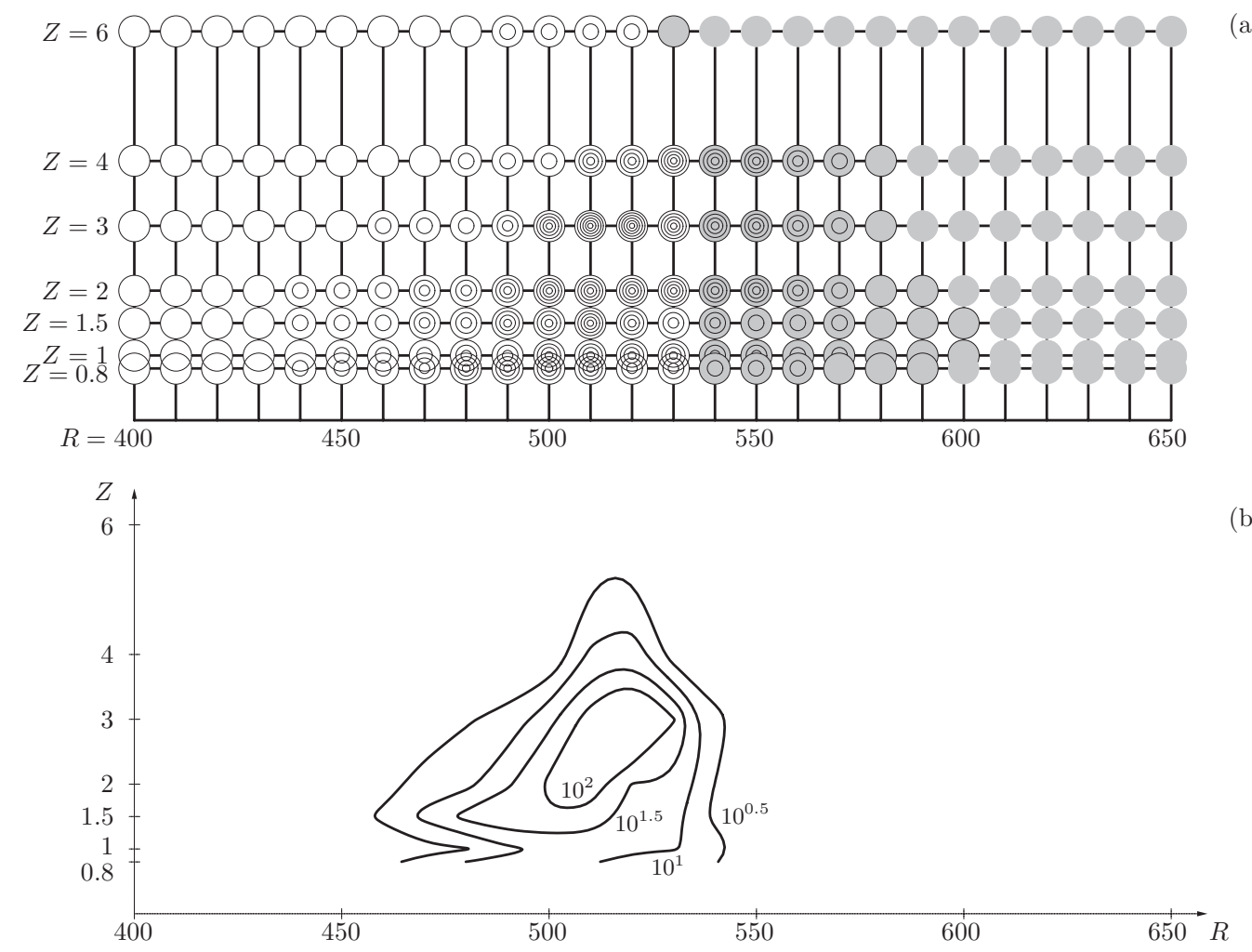

(a)

FIG. 9. (a) Detailed map of the local boundary-layer features, based on the low-resolution spectra recorded at $R=400$, $410, \ldots, 650$ and $Z=0.8,1,1.5,2,3,4$, and 6 . The symbols depict the type of spectrum that prevails at a given location: the number of circles corresponds to the number of harmonic peaks that could be identified and a grey background indicates the presence of a broadband component. (b) Contour plot measuring the importance of the discrete component in the high-resolution velocity spectra. The measure is based on the mean ratio between the integer-frequency maxima and the semi-integer-frequency minima of the high-resolution spectra.

important, another post-processing script has been developed: for each of the high-resolution spectra, the script extracts the maxima located at integer frequencies and the minima located at half-integer frequencies, and then computes the average separation of these alternating extrema, on a logarithmic scale. The result represents a measure of the amplitude of the discrete spectral component and is shown as a contour plot with equispaced iso-levels in the $(R, Z)$-plane in Figure 9(b). Comparing Figures 9(a) and 9(b) clearly demonstrates that the presence of this discrete component coincides with the existence of a weakly nonlinear regime. Moreover, the discrete component has maximal strength around $R=520$ and $Z=3$, which is also where the largest number of harmonic peaks have been identified. It should also be observed that this radial position nearly coincides with the onset of local absolute instability at $R=507$. To our knowledge, despite much previous research on the rotating-disk boundary-layer flow, the only previous discussion of the discrete part of the velocity spectra is to be found in Refs. 13 and 14, though it is visible in the results of Ref. 15. One reason for the poor documentation of this feature may be the fact that it is only observable using high-resolution spectra and thus requires measurement of velocity time-series over long time periods.

Traditionally, the rotating-disk boundary layer is considered as an example of a flow exhibiting a transition from the laminar to the turbulent regime much shorter than the case for other boundary layers, such as the flat plate boundary layer. However, measurements close to the disk surface indicate that an intermediate nonlinear regime is present over a considerable range of $R$. In a recent study, Imayama et al. ${ }^{12}$ have characterized the evolution of the boundary layer by measuring the probability density function of the fluctuating azimuthal disturbance velocity. In the present investigation, by systematically acquiring low- and high-resolution spectra over a range of narrowly spaced radial and 
axial positions, further light is shed on the detailed structure of the complex transition region close to the disk surface.

\section{ACKNOWLEDGMENTS}

The invaluable technical assistance by Alexandre Azouzi, Horacio Correia, Roger Michelet, Christian Nicot, and Bruno Poirel is gratefully acknowledged.

This research was financially supported by Agence nationale de la recherche (Project "Microsillon").

${ }^{1}$ Th. von Kármán, “Über laminare und turbulente Reibung,” Z. Angew. Math. Mech. 1, 233-252 (1921).

${ }^{2}$ N. Gregory, J. T. Stuart, and W. S. Walker, "On the stability of three-dimensional boundary layers with application to the flow due to a rotating disk," Philos. Trans. R. Soc. London, Ser. A 248, 155-199 (1955).

${ }^{3}$ R. Kobayashi, Y. Kohama, and C. Takamadate, "Spiral vortices in boundary layer transition regime on a rotating disk," Acta Mechanica 35, 71-82 (1980).

${ }^{4}$ M. R. Malik, S. P. Wilkinson, and S. A. Orszag, "Instability and transition in rotating disk flow,” AIAA J. 19, 1131-1138 (1981).

${ }^{5}$ S. P. Wilkinson and M. R. Malik, "Stability experiments in the flow over a rotating disk," AIAA J. 23, 588-595 (1985).

${ }^{6}$ R. J. Lingwood, “An experimental study of absolute instability of the rotating-disk boundary-layer flow," J. Fluid Mech. 314, 373-405 (1996).

${ }^{7}$ H. Othman and T. Corke, "Experimental investigation of absolute instability of a rotating-disk boundary layer," J. Fluid Mech. 565, 63-94 (2006).

${ }^{8}$ R. J. Lingwood, "Absolute instability of the boundary layer on a rotating disk," J. Fluid Mech. 299, 17-33 (1995).

${ }^{9}$ C. Davies and P. W. Carpenter, "Global behaviour corresponding to the absolute instability of the rotating-disk boundary layer," J. Fluid Mech. 486, 287-329 (2003).

${ }^{10}$ B. Pier, "Finite-amplitude crossflow vortices, secondary instability and transition in the rotating-disk boundary layer," J. Fluid Mech. 487, 315-343 (2003).

${ }^{11}$ J. J. Healey, "Model for unstable global modes in the rotating-disk boundary layer," J. Fluid Mech. 663, 148-159 (2010).

${ }^{12}$ S. Imayama, P. H. Alfredsson, and R. J. Lingwood, "A new way to describe the transition characteristics of a rotating-disk boundary-layer flow," Phys. Fluids 24, 031701 (2012).

${ }^{13}$ M. E. Siddiqui, "Experimental study of natural and forced instabilities and transition of a rotating-disk boundary-layer flow," Ph.D. dissertation (École centrale de Lyon, Écully, France, 2011).

${ }^{14}$ M. E. Siddiqui, B. Pier, J. Scott, A. Azouzi, and R. Michelet, "Experimental characterization and control of transition region in rotating-disk boundary layer," in Seventh IUTAM Symposium on Laminar-Turbulent Transition, edited by P. Schlatter and D. S. Henningson (Springer, Stockholm, Sweden, 2010), pp. 573-576.

${ }^{15}$ S. Imayama, P. H. Alfredsson, and R. J. Lingwood, "An experimental study of laminar-turbulent transition of a rotating-disk flow," in EUROMECH Colloquium 525, Instabilities and Transition in Three-Dimensional Flows with Rotation (Écully, France, 2011), pp. 29-30, see http://hal.archives-ouvertes.fr/EC525/hal-00600333. 